\title{
Optimizing Pinhole and Parallel Hole Collimation for Scintimammography With Compact Pixellated Detectors
}

\author{
Mark F. Smith, Member, IEEE, Stan Majewski, and Andrew G. Weisenberger
}

\begin{abstract}
The relative resolution and sensitivity advantages of pinhole and parallel hole collimators for planar scintimammography with compact, pixellated gamma detectors were investigated using analytic models. Collimator design was studied as follows. A desired object resolution was specified for a pixellated detector with a given crystal size and intrinsic spatial resolution and for a given object-to-collimator distance. Using analytic formulas, pinhole and parallel hole collimator parameters were calculated that satisfy this object resolution with optimal geometric sensitivity. Analyses were performed for $15 \mathrm{~cm} \times 20 \mathrm{~cm}$ field of view detectors with crystal elements $1.0,2.0$, and $3.0 \mathrm{~mm}$ on a side and $140 \mathrm{keV}$ incident photons. The sensitivity for a given object resolution was greater for pinhole collimation at smaller distances, as expected. The object distance at which the pinhole and parallel hole sensitivity curves cross each other is important. The crossover distances increased with larger crystal size for a constant object resolution and increased as the desired object resolution decreased for a constant crystal size. For example, for $4 \mathrm{~mm}$ object resolution and a pinhole collimator with focal length $13 \mathrm{~cm}$, these distances were $5.5 \mathrm{~cm}, 6.5 \mathrm{~cm}$, and $8 \mathrm{~cm}$ for the $1 \mathrm{~mm}, 2 \mathrm{~mm}$, and $3 \mathrm{~mm}$ crystal detectors, respectively. The results suggest a strategy of parallel hole collimation for whole breast imaging and pinhole collimation for imaging focal uptake. This could be accomplished with a dual detector system with a different collimator type on each head or a single head system equipped with two collimators and a rapid switching mechanism.
\end{abstract}

Index Terms-Collimator, collimator optimization, parallel hole collimator, pinhole collimator, pixellated detector, scintimammography.

\section{INTRODUCTION}

$\mathbf{P}$ ARALLEL hole collimation [1] and pinhole collimation [2], [3] have been employed for scintimammography with standard gamma cameras. Pinhole collimation with object magnification permits image resolution that is better than the intrinsic resolution of the detector. Sensitivity is improved when the pinhole is moved closer to the object being imaged, at the expense of a smaller field of view. Resolution with parallel hole collimation degrades as the source to collimator distance increases, while the sensitivity and field of view remain approx-

Manuscript received December 6, 2002; revised February 28, 2003. This work was supported by the Office of Biological and Environmental Research, Office of Science of U.S. Department of Energy. The Southeastern Universities Research Association (SURA) operates the Thomas Jefferson National Accelerator Facility for the U.S. Department of Energy under Contract DE-AC05-84ER40150.

The authors are with the Thomas Jefferson National Accelerator Facility, Newport News, VA 23606 USA (e-mail: mfsmith@jlab.org; majewski@jlab.org; drew@jlab.org).

Digital Object Identifier 10.1109/TNS.2003.812436 imately constant as a function of distance. Pinhole collimation is generally advantageous for high resolution, high sensitivity imaging of small objects, while parallel hole collimation generally has the sensitivity advantage where resolution requirements are not as stringent and where larger objects must be imaged.

We and others have previously investigated the application of compact, pixellated detectors with parallel hole collimation for scintimammography [4]-[8] and a recent clinical study has demonstrated some advantages for detecting small $(<1 \mathrm{~cm})$ lesions compared with a conventional gamma camera [9]. These compact detectors also can be equipped with pinhole collimators and so we seek a quantitative understanding of the relative resolution and sensitivity advantages of optimally designed pinhole and parallel hole collimators for planar breast imaging.

Imaging characteristics of parallel hole and pinhole collimators for scintimammography a conventional gamma camera have been compared [10], though collimator optimization was not evaluated. Monte Carlo modeling has been used to study the performance of compact gamma cameras with discretized scintillation crystal arrays [11]. Various types of orbits for SPECT scintimammography also have been an active area of recent investigation [12]-[14].

In this contribution the design of pinhole and parallel hole collimators for planar breast imaging is investigated as follows. A desired object resolution is specified for a pixellated detector with a given crystal size and intrinsic spatial resolution, and for a given object-to-collimator distance. Then, using analytic formulas, the pinhole and parallel hole collimator parameters are calculated that meet this object resolution with optimal geometric sensitivity. The goal of this analysis is to provide insight into collimator design and selection tradeoffs for clinical studies.

\section{METHODS}

\section{A. Pinhole Collimator Optimization}

Analytic formulas for the resolution and geometric sensitivity of pinhole collimators have been previously developed [15]. Object resolution in the image is given by

$$
R_{o}=\left(\frac{x}{f}\right)\left[R_{D}^{2}+\left(\frac{f+x}{x}\right)^{2} d_{e}^{2}\right]^{1 / 2}
$$

and geometric point sensitivity by

$$
g=d_{e}^{2} \frac{\cos ^{3} \theta}{16 x^{2}}
$$


with the effective pinhole diameter

$$
d_{e}=\left[d\left(d+\frac{2}{\mu} \tan \frac{\alpha}{2}\right)\right]^{1 / 2}
$$

Here, $f$ is the focal length, $x$ is the distance from the pinhole to a point source along the central axis of the collimator, $\theta$ is the raypath angle from the central axis, $R_{D}$ is the intrinsic spatial resolution of the detector, $d$ is the pinhole diameter, $\mu$ is the attenuation coefficient of the pinhole material, and $\alpha$ is the full acceptance angle of the aperture [Fig. 1(a) and (b)]. When $x, f$, and $R_{D}$ are set, $d_{e}$ can be varied to achieve a particular object resolution $R_{O}$ (1) and then the geometric point sensitivity can be computed using (2). The explicit equation for sensitivity is then

$$
g=\frac{\cos ^{3} \theta}{16(x+f)^{2}}\left[\left(\frac{f}{x}\right)^{2} R_{o}^{2}-R_{D}^{2}\right]
$$

\section{B. Parallel Hole Collimator Optimization}

Optimization of geometric sensitivity for a parallel hole collimator for a given object resolution can be performed by the approach of Keller [16] that uses the sensitivity and resolution formulas of Anger [15]. In brief, geometric sensitivity can be expressed by

$$
g=\left[\frac{K d^{2}}{a_{e}(d+t)}\right]^{2}
$$

where $K$ is a collimator hole shape factor, $d$ is the hole width, $t$ is the septal thickness, and the effective hole length $a_{e}$ is given by

$$
a_{e}=a-\frac{2}{\mu}
$$

where $a$ is the physical hole length and $\mu$ is the attenuation coefficient of the collimator material [Fig. 1(c)].

The object resolution can be expressed by

$$
R_{o}=\left[R_{D}^{2}+R_{g}^{2}\right]^{1 / 2}
$$

where $R_{D}$ is the intrinsic spatial resolution of the detector as for the pinhole camera and the geometric resolution is

$$
R_{g}=\frac{d\left(a_{e}+x+c\right)}{a_{e}}
$$

Here, $x$ is the object to collimator distance and $c$ is the distance from the end of the collimator to the middle of the scintillation crystal.

The minimal raypath distance in the septa can be written $w=-(\ln \beta) / \mu$ where $\beta$ is the maximum allowable septal penetration factor. The septal thickness $t$ then can be approximated by

$$
t=\frac{2 d w}{a-w}
$$

When $R_{o}, R_{D}, x$, and $c$ are set, (6) can be substituted into (5), (8) can be solved for $d$ and substituted into (5) and (9), and then the expression for $t$ can be substituted into (5). The

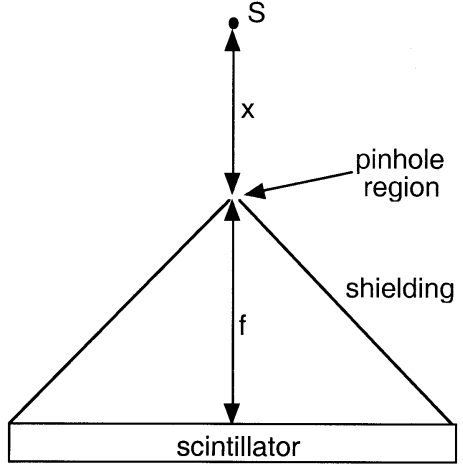

(a)

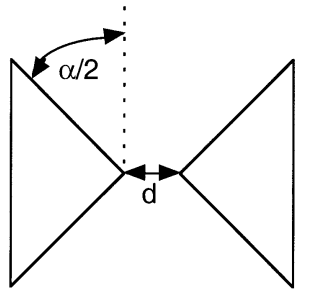

(b)

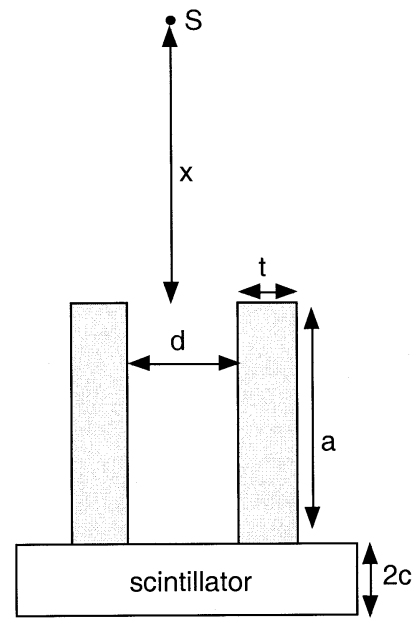

(c)

Fig. 1. Diagrams of cross sections through: (a) a pinhole collimator and (b) a close-up of the pinhole aperture region. A point source $S$ is a distance $x$ from the pinhole along the central axis of the collimator, $f$ is the focal length, $d$ is the pinhole diameter of a knife-edge collimator, and $\alpha$ is the full acceptance angle. (c) Diagram of a section of a parallel hole collimator with a point source $S$ a distance $x$ from the collimator surface. Here $d$ is the collimator hole width, $t$ is the septal thickness, $a$ is the collimator hole length, and $c$ is the distance from the front scintillator surface to the scintillator midplane.

derivative with respect to hole length $a$ can be taken, which leads to maximal sensitivity when

$$
a=-\frac{\ln \beta}{\mu}+\left[2\left(\frac{\ln \beta}{\mu}\right)^{2}+4 \frac{\ln \beta}{\mu^{2}}-2(x+c) \frac{\ln \beta}{\mu}\right]^{1 / 2} .
$$

This is a minor generalization of the result in [16]. Once the optimal value of $a$ is known, one can solve for $a_{e}(6), d(8), t$ (9) and $g(5)$. An explicit equation for sensitivity is

$$
g=\left[\frac{K\left(R_{o}^{2}-R_{D}^{2}\right)^{1 / 2}\left(a+\frac{\ln \beta}{\mu}\right)}{\left(a-\frac{2}{\mu}+x+c\right)\left(a-\frac{\ln \beta}{\mu}\right)}\right]^{2}
$$


with distance $(x)$-dependent $a$ as in (10).

\section{Comparison of Pinhole and Parallel Hole Collimation}

Calculations were performed for a pixellated detector with a $15 \mathrm{~cm} \times 20 \mathrm{~cm}$ field of view and for $140 \mathrm{keV}$ incident photons. Optimized collimator parameters were computed for detectors with crystal elements 1.0, 2.0, and $3.0 \mathrm{~mm}$ on a side, $5 \mathrm{~mm}$ long and with crystal pitches $1.25,2.25$, and $3.25 \mathrm{~mm}$, respectively. Detector resolution was modeled to be the same as the crystal pitch. The effect of the $0.25 \mathrm{~mm}$ optical diffusing material between the crystals was included in the sensitivity calculations.

For pinhole breast imaging two sets of sensitivities were computed. For set I, the collimator focal length was chosen with the requirement that the sensitivity $10 \mathrm{~cm}$ from the collimator axis for a magnification factor of 1.0 be at least $50 \%$ of the sensitivity on the collimator axis. The $10 \mathrm{~cm}$ distance is at one edge of a $15 \mathrm{~cm} \times 20 \mathrm{~cm}$ detector for a centered pinhole. This leads to a minimum focal length of $13.0 \mathrm{~cm}$; at smaller focal lengths the angular dependence of sensitivity (2) leads to relative sensitivity values of less than one-half.

For set II the focal length was chosen with the requirement that the entire (compressed) breast lie within the field of view of the imager. For a given breast with compressed diameter $D_{b}$ and with maximal distance $(1 / 2) D_{b}$ from the central pinhole axis, the distance $x$ from the pinhole was minimized while maintaining the requirement that the sensitivity at the edge of the breast be no less than $50 \%$ of the sensitivity at a point on the central axis. This requirement leads to $x=(1 / 2) D_{b} \cot \left(\arccos \left(0.5^{1 / 3}\right)\right)=(0.652) D_{b}$. Next the focal length $f$ was set so that the breast image would fill the field of view of the detector and thus achieve the best image resolution (1). This leads to $f=(15 \mathrm{~cm})\left(x / D_{b}\right)=9.79 \mathrm{~cm}$. Since the maximum angle from the central pinhole axis for a point in the breast that meets the above sensitivity requirement is $\arccos \left(0.5^{1 / 3}\right)=37.5^{\circ}$, it follows that the maximum diameter breast that can be viewed at any distance $x$ from the collimator with the above sensitivity requirement is $D_{b}=2 x \tan \left(\arccos \left(0.5^{1 / 3}\right)\right)=1.53 x$.

Results are presented for 3.0 and $4.0 \mathrm{~mm}$ object resolutions in the images for object-to-collimator distances between 1 and $10 \mathrm{~cm}$. The pinhole collimator was assumed to be fabricated with an $18.0 \mathrm{~g} / \mathrm{cm}^{3}$ tungsten alloy and sensitivities were computed on the collimator axis. The parallel hole collimator was assumed to be lead with square holes in a square grid $(K=0.282)$ [15] and the allowable septal penetration factor $\beta$ was 0.05 .

\section{RESULTS}

Graphs of sensitivity vs. object distance from the pinhole or collimator surface are shown in Figs. 2 and 3. The pinhole collimator curves in Fig. 2 are for a collimator with a focal length of $13 \mathrm{~cm}$, while those in Fig. 3 are for a collimator with a focal length of $9.79 \mathrm{~cm}$. The parallel hole collimator curves are the same in both figures. Note that each individual point in these curves represents the sensitivity value for different collimator parameters that meet the specified resolution condition and, for pinhole collimation, the fixed focal length condition indicated in the graph titles. For the parallel hole collimator, for example,
Sensitivity vs. Distance

$1.25 \mathrm{~mm}$ Detector Resolution, $\mathrm{f}=13 \mathrm{~cm}$

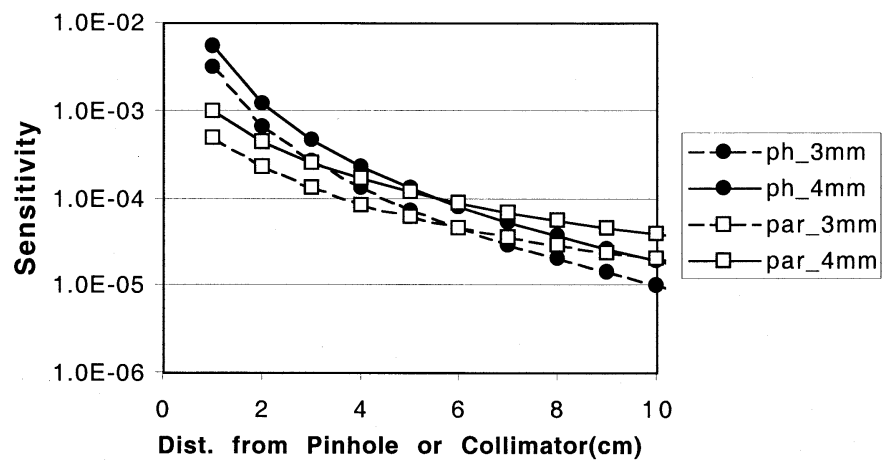

(a)

Sensitivity vs. Distance

$2.25 \mathrm{~mm}$ Detector Resolution, $f=13 \mathrm{~cm}$

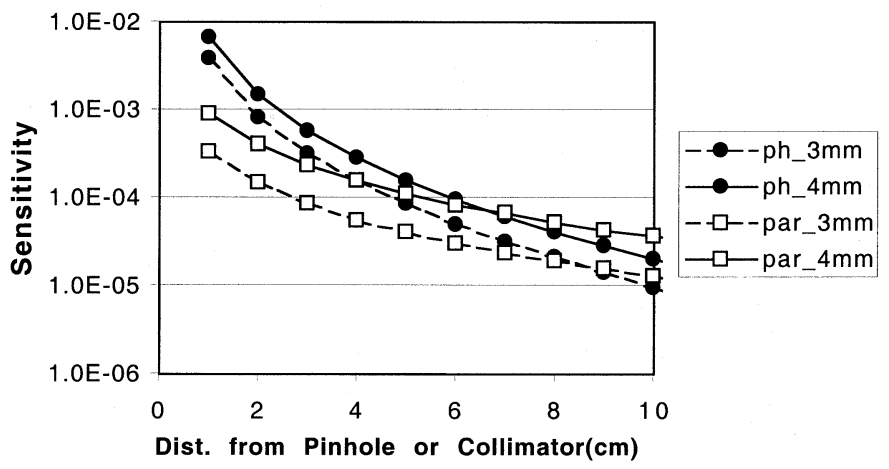

(b)

Sensitivity vs. Distance

$3.25 \mathrm{~mm}$ Detector Resolution, $f=13 \mathrm{~cm}$

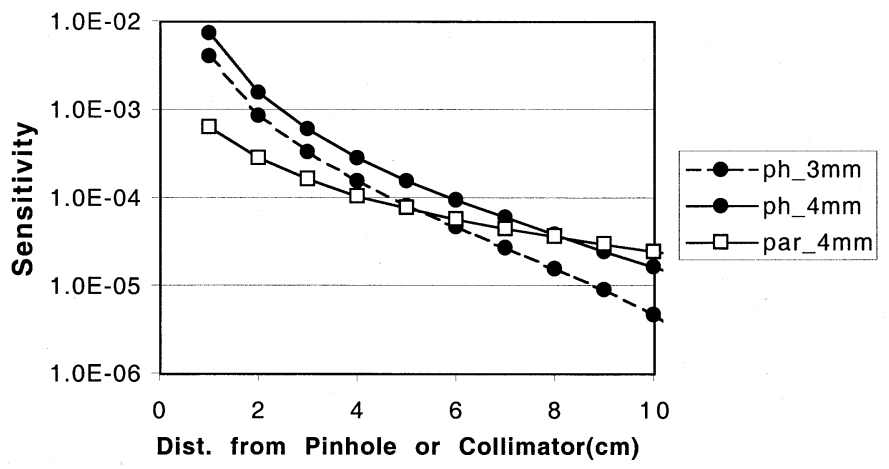

(c)

Fig. 2. Geometric sensitivity versus object-collimator distance for optimized pinhole and parallel collimators, and pixellated detectors with intrinsic spatial resolutions of: (a) $1.25 \mathrm{~mm}$, (b) $2.25 \mathrm{~mm}$, and (c) $3.25 \mathrm{~mm}$. The focal length $f$ of the pinhole collimator is fixed at $13 \mathrm{~cm}$. Legend notation: $\mathrm{ph}=$ pinhole, $\mathrm{par}=$ parallel hole, followed by the desired object resolution in the image. Note that the collimator parameters are different at each distance (see Section II).

this results in the sensitivity decreasing with object distance from the collimator surface as the collimator hole dimensions are adjusted in order to meet the specified resolution requirement.

As expected, sensitivity for a given object resolution is greater for pinhole collimation for smaller object distances. The distance at which the pinhole and parallel hole curves cross each other is important. For the pinhole collimator with focal length $13 \mathrm{~cm}$, the crossover distance for $1.25 \mathrm{~mm}$ detector resolution 
Sensitivity vs. Distance $1.25 \mathrm{~mm}$ Detector Resolution, $f=9.79 \mathrm{~cm}$

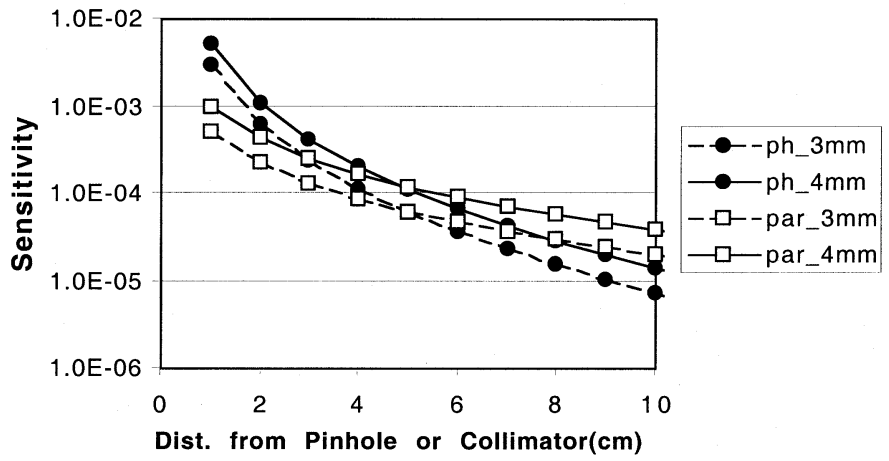

(a)

Sensitivity vs. Distance

$2.25 \mathrm{~mm}$ Detector Resolution, $\mathrm{f}=9.79 \mathrm{~cm}$

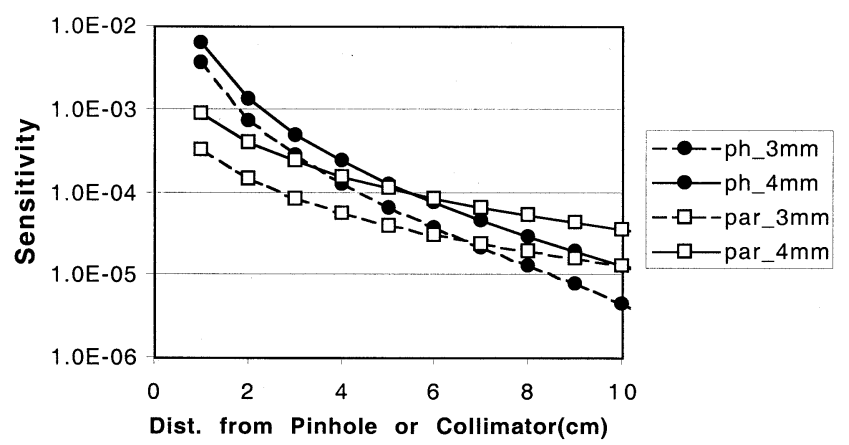

(b)

Sensitivity vs. Distance

$3.25 \mathrm{~mm}$ Detector Resolution, $f=9.79 \mathrm{~cm}$

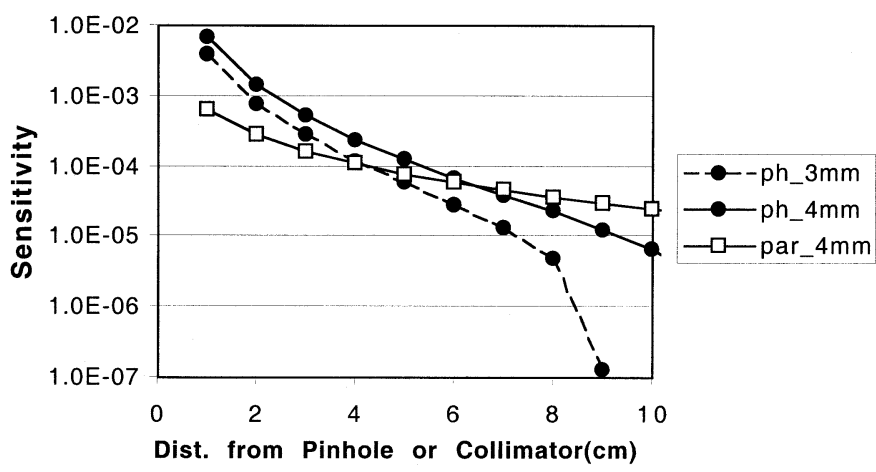

(c)

Fig. 3. Geometric sensitivity versus object- collimator distance for optimized pinhole and parallel collimators, and pixellated detectors with intrinsic spatial resolutions of (a): $1.25 \mathrm{~mm}$, (b) $2.25 \mathrm{~mm}$, and (c) $3.25 \mathrm{~mm}$. The focal length $f$ of the pinhole collimator is $9.79 \mathrm{~cm}$. The maximum diameter (compressed) breast entirely within the field of view of the gamma camera at a distance $x$ from the pinhole collimator meeting the desired off-axis sensitivity requirements is 1.53x. Legend notation: $\mathrm{ph}=$ pinhole, $\mathrm{par}=$ parallel hole, followed by the desired object resolution in the image. Note that the collimator parameters are different at each distance (see Section II).

is $5.5 \mathrm{~cm}$ for $4 \mathrm{~mm}$ object resolution and $6 \mathrm{~cm}$ for $3 \mathrm{~mm}$ object resolution; for $2.25 \mathrm{~mm}$ detector resolution the crossover distance is $6.5 \mathrm{~cm}$ for $4 \mathrm{~mm}$ object resolution and $8.5 \mathrm{~cm}$ for $3 \mathrm{~mm}$ object resolution; for $3.25 \mathrm{~mm}$ detector resolution the crossover distance is $8 \mathrm{~cm}$ for $4 \mathrm{~mm}$ object resolution and $3 \mathrm{~mm}$ object resolution cannot be achieved with parallel hole collimation. The crossover distances: 1) increase with larger crystal size for a

\section{Effective Pinhole Diameter $(\mathrm{f}=13 \mathrm{~cm})$}

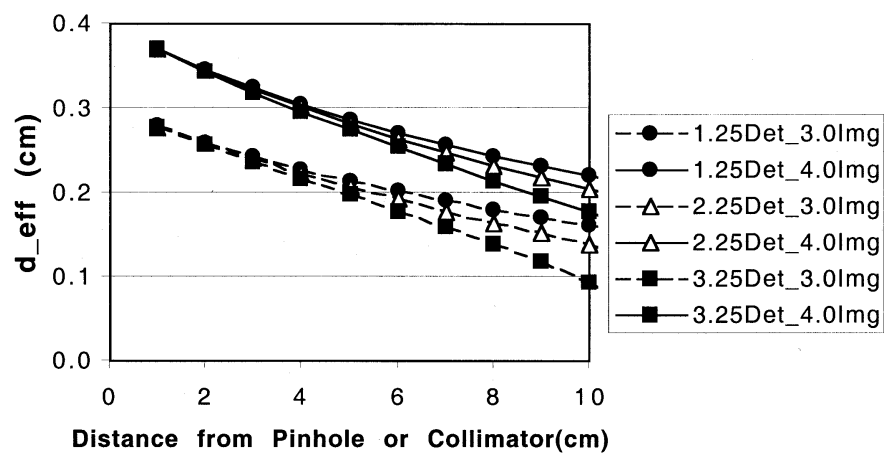

(a)

Effective Pinhole Diameter $(f=9.79 \mathrm{~cm})$

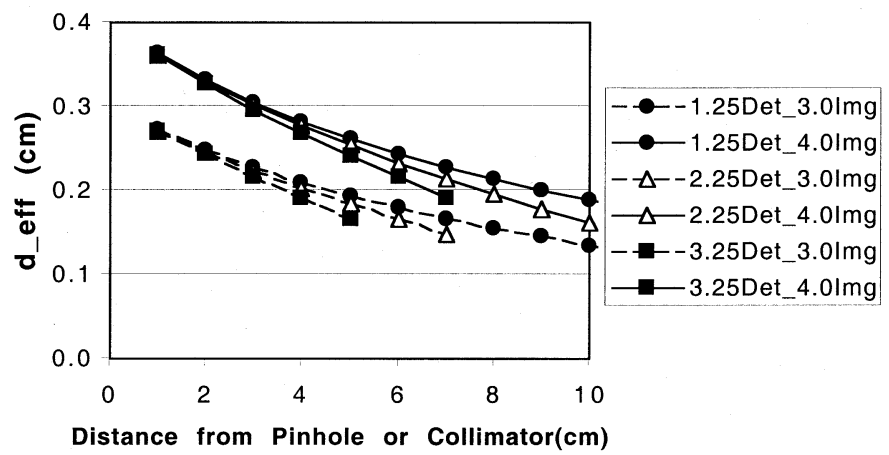

(b)

Fig. 4. Effective pinhole diameters for pinhole collimators with focal lengths: (a) $13 \mathrm{~cm}$ and (b) $9.79 \mathrm{~cm}$. Legend notation: detector resolution $(\mathrm{mm})$ followed by the desired object resolution in the image $(\mathrm{mm})$.

constant object resolution and 2) increase as the desired object resolution decreases for a constant crystal size.

The crossover distances are all smaller for the $9.79 \mathrm{~cm}$ focal length pinhole collimator than for the $13 \mathrm{~cm}$ focal length collimator. This is expected since, for a given object distance from the pinhole aperture, magnification is not as great for $f=9.79 \mathrm{~cm}$ as for $f=13 \mathrm{~cm}$. In fact, minification will occur when the point of interest in the breast is farther than the focal length from the pinhole. The pinhole aperture to achieve a desired image resolution is smaller for $f=9.79 \mathrm{~cm}$, leading to reduced sensitivity, which limits the advantageous region for pinhole collimation.

The effective pinhole diameters for the pinhole collimators are shown in Fig. 4. The trends of these curves are in accord with expectations: the effective diameter decreases with increasing distance of the imaged point from the pinhole aperture, decreases as the intrinsic detector resolution increases, is larger for a greater image resolution, and is smaller when the collimator focal length is reduced.

Optimized parallel hole collimator parameters are displayed in Fig. 5. The hole length, $a$, increases with object distance from the collimator surface [Fig. 5(a)] and is independent of intrinsic detector resolution and desired object resolution (10). The hole width and septal thickness decrease as a function of distance, decrease as the intrinsic detector resolution increases, and decrease as the desired image resolution decreases. These changes 
Optimized Hole Length, a

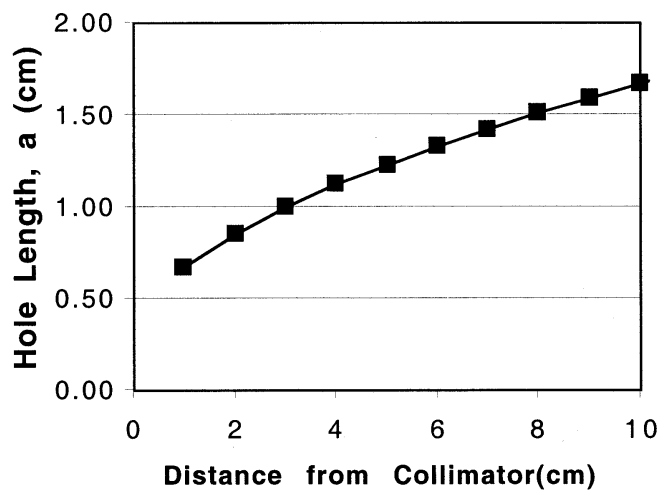

(a)

Optimized Hole Width, d

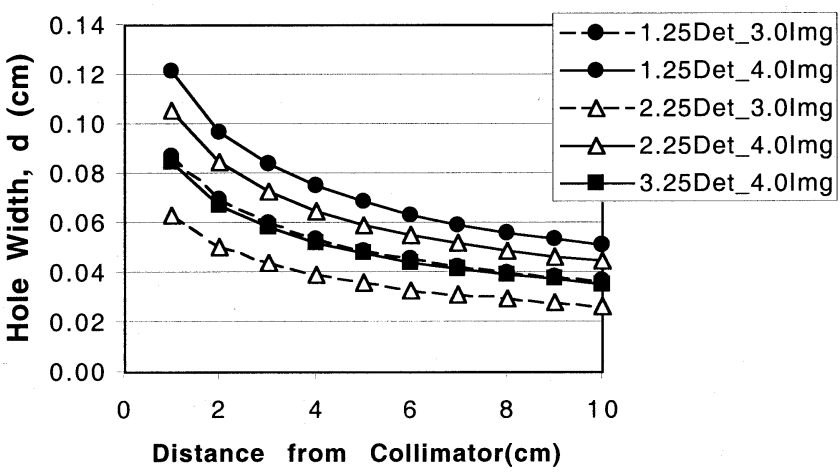

(b)

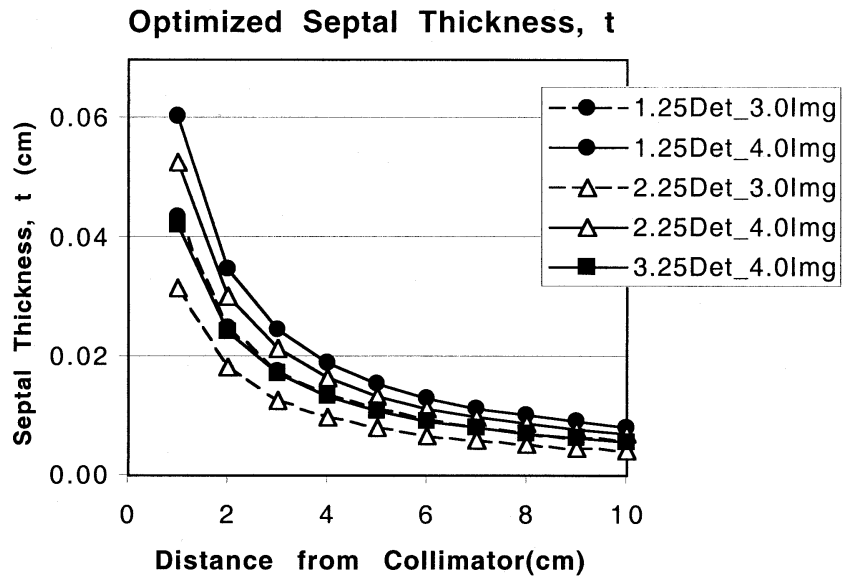

(c)

Fig. 5. Optimized parallel hole collimator: (a) hole length, (b) hole width, and (c) septal thickness. Legend notation: detector resolution $(\mathrm{mm})$ followed by the desired object resolution in the image $(\mathrm{mm})$. In parts (b) and (c), the curves 3.25Det_4.0Img and 1.25Det_3.0Img almost overlay each other.

in parameters as the object-to-collimator distance decreases result in improved sensitivity at closer distances (Figs. 2 and 3).

\section{Discussion}

For imaging an entire compressed breast ( $\sim 4-6 \mathrm{~cm}$ thick) with good sensitivity near the edge of the field of view, parallel hole collimation usually will be the best option. The front face of the collimator can be placed directly above the compression paddle to minimize the distance between the breast tissue and the collimator. Sensitivity will be better than can be achieved with a pinhole collimator whose aperture must be placed a greater distance from the breast in order to image the entire breast in the field of view of the detector. On the other hand, for imaging suspicious activity uptake in just part of the breast, the pinhole can be brought close to the breast and greater sensitivity can be achieved than with parallel hole collimation.

The above results provide a strategy for the efficient use of compact pixellated detectors for scintimammography: parallel hole collimation for whole breast imaging and pinhole collimation for high resolution, high sensitivity imaging of anomalous activity regions. This could be accomplished with a dual detector system with a different type of collimator on each head or with a single head system with a rapid collimator switching mechanism. If pinhole imaging of the whole breast is desired, then the design, fabrication and use of a collimator with a mechanically variable focal length may be advantageous.

A $50 \%$ off-axis sensitivity threshold was used for selecting the focal length of the pinhole collimators studied in this paper. This yielded two different focal lengths depending on additional requirements regarding magnification and imaging the entire breast. Different criteria could be used that would result in different focal lengths. Similar analyses of the relative strengths of pinhole and parallel hole collimation could then be performed.

This comparison of parallel hole and pinhole collimators has been made using analytic formulas and is an initial effort toward understanding the relative resolution and sensitivity advantages of parallel hole and pinhole collimation for scintigraphic imaging with compact, pixellated detectors. A fair comparison of the advantages between collimator types must consider optimization of the collimator parameters rather than just evaluating a few selected designs of each collimator type. The experimental comparison of examples of existing physical pinhole or parallel hole collimators is a separate evaluation task.

In the future, the more accurate analytic expressions for sensitivity and point spread functions of pinhole collimators of Metzler et al. [17], [18] could be used to provide more refined tradeoff curves. A more comprehensive but more computationally demanding analysis could include raytracing or Monte Carlo simulation of planar and SPECT acquisition. For pinhole collimation channeled pinhole apertures [19] as well as knife-edge apertures should be modeled, and scatter and within the source region as well as within the pinhole attenuating material should be considered. Scatter and penetration within a parallel hole collimator could be modeled as well [20], [21]. For a detector with a pixellated scintillator, a more accurate model of acquisition would include modeling of photon transport within the scintillator array.

A task-based collimator evaluation could include image reconstruction for SPECT and an investigation of contrast and signal-to-noise tradeoffs for different lesion sizes and tumor: background activity concentration ratios. If planar image resolution is the same for two different collimator types, then contrast (at least along the central pinhole axis) should be approximately the same and the collimator with greater sensitivity 
should provide a signal-to-noise advantage. The results in this paper may be useful in collimator design or selection for small animal imaging with compact pixellated detectors.

\section{CONCLUSION}

The relative resolution and sensitivity advantages of pinhole and parallel hole collimators for planar scintimammography with compact pixellated gamma detectors were investigated using analytic formulas. For a $15 \mathrm{~cm} \times 20 \mathrm{~cm}$ field of gamma camera with pixellated scintillators having crystals $1-3 \mathrm{~mm}$ on a side, sensitivity for a given object resolution was greater for pinhole collimation at smaller distances, as expected. The crossover distances between the pinhole and parallel hole sensitivity curves increased with larger crystal size for a constant object resolution and increased as the desired object resolution decreases for a constant crystal size. The results suggest a strategy of parallel hole collimation for whole breast imaging and pinhole collimation for imaging focal uptake.

\section{REFERENCES}

[1] I. Khalkhali, J. Cutrone, I. Mena, L. Diggles, R. Venegas, H. Vargas, B. Jackson, and S. Klein, "Technetium-99m-sestamibi scintimammography of breast lesions: Clinical and pathological follow-up," J. Nucl. Med., vol. 36, pp. 1784-1789, 1995.

[2] C. Scarfone, R. J. Jaszczak, J. Li, M. S. Soo, M. F. Smith, K. L. Greer, and R. E. Coleman, "Breast tumor imaging using incomplete circular orbit pinhole SPECT: A phantom study," Nucl. Med. Commun., vol. 18, pp. 1077-1086, 1997.

[3] A. Spanu, G. Dettori, P. Chiaramida, P. Cottu, A. Falchi, A. Porcu, S. M. E., S. Nuvoli, and G. Madeddu, "The role of $99 \mathrm{~m} T c$-tetrofosmin pinhole-SPECT in breast cancer axillary lymph node staging," Cancer Biother. Radiopharm., vol. 15, pp. 81-91, 2000.

[4] R. Pani, G. De Vincentis, F. Scopinaro, R. Pellegrini, A. Soluri, I. N. Weinberg, A. Pergola, R. Scafè, and G. Trotta, "Dedicated gamma camera for single photon emission mammography (SPEM)," IEEE Trans. Nucl. Sci., vol. 45, pp. 3127-3133, 1998.

[5] C. L. Maini, F. de Notaristefani, A. Tofani, F. Iacopi, R. Sciuto, A. Semprebene, T. Malatesta, F. Vittori, F. Frezza, C. Botti, S. Giunta, and P. G. Natali, " $99 m T c$-MIBI scintimammography using a dedicated nuclear mammograph," J. Nucl. Med., vol. 40, pp. 46-51, 1999.

[6] D. P. McElroy, E. J. Hoffman, M. L., B. E. Patt, J. S. Iwanczyk, Y. Yamaguchi, and C. S. Levin, "Evaluation of performance of dedicated, compact scintillation cameras," in Conf. Rec. 2000 IEEE Nuclear Science Symp. Medical Imaging, 2000, pp. 21-109-21-113.
[7] M. B. Williams, A. R. Goode, V. Galbis-Reig, S. Majewski, A. G. Weisenberger, and R. Wojcik, "Performance of a PSPMT based detector for scintimammography," Phys. Med. Biol., vol. 45, pp. 781-800, 2000.

[8] S. Majewski, D. Kieper, E. Curran, C. Keppel, B. Kross, A. Palumbo, V. Popov, A. G. Weisenberger, B. Welch, R. Wojcik, M. B. Williams, A. R. Goode, M. More, and G. Zhang, "Optimization of dedicated scintimammography procedure using detector prototypes and compressible phantoms," IEEE Trans. Nucl. Sci., vol. 48, pp. 822-829, 2001.

[9] R. F. Brem, J. M. Schoonjans, D. A. Kieper, S. Majewski, S. Goodman, and C. Civelek, "High-resolution scintimammography: A pilot study," J. Nucl. Med., vol. 43, pp. 909-915, 2002.

[10] B. M. W. Tsui, D. E. Wessell, X. D. Zhao, W. T. Wang, D. P. Lewis, and E. C. Frey, "Imaging characteristics of scintimammography using parallel-hole and pinhole collimators," IEEE Trans. Nucl. Sci., vol. 45, pp. 2155-2161, 1998.

[11] G. J. Gruber, W. W. Moses, and S. E. Derenzo, "Monte Carlo simulation of breast tumor imaging properties with compact, discrete gamma cameras," IEEE Trans. Nucl. Sci., vol. 46, pp. 2119-2123, 1999.

[12] H. Wang, C. Scarfone, K. L. Greer, R. E. Coleman, and R. J. Jaszczak, "Prone breast tumor imaging using vertical axis-of-rotation (VAOR) SPECT systems: An initial study," IEEE Trans. Nucl. Sci., vol. 44, pp. 1271-1276, 1997.

[13] M. P. Tornai, J. E. Bowsher, C. N. Archer, J. Peter, L. R. MacDonald, B. E. Patt, J. S. Iwanczyk, R. J. Jaszczak, and R. E. Coleman, "A novel application specific emission tomograph (ASET) for breast imaging," in Conf. Rec. 2001 IEEE Nuclear Science Symp. Medical Imaging, 2001, pp. 1161-1165.

[14] S. D. Metzler, J. E. Bowsher, M. P. Tornai, B. C. Pieper, J. Peter, and R. J. Jaszczak, "SPECT breast imaging combining horizontal and vertical axes of rotation," IEEE Trans. Nucl. Sci., vol. 49, pp. 31-36, Feb. 2002.

[15] H. O. Anger, Radioisotope Cameras in Instrumentation in Nuclear Medicine, G. J. Hine, Ed. New York: Academic, 1967, vol. 1, pp. 485-552.

[16] E. L. Keller, "Optimum dimensions of parallel-hole, multi-aperture collimators for gamma-ray cameras," J. Nucl. Med., vol. 9, pp. 233-235, 1968.

[17] S. D. Metzler, J. E. Bowsher, M. F. Smith, and R. J. Jaszczak, "Analytic determination of pinhole collimator sensitivity with penetration," IEEE Trans. Med. Imaging, vol. 20, pp. 730-741, 2001.

[18] S. D. Metzler, J. E. Bowsher, K. L. Greer, and R. J. Jaszczak, "Analytic determination of the pinhole collimator's point-spread function and RMS resolution with penetration," in Conf. Rec. 2001 IEEE Nuclear Science Symp. Medical Imaging, 2001, pp. 1175-1179.

[19] M. F. Smith, R. J. Jaszczak, H. Wang, and J. Li, "Lead and tungsten pinhole inserts for I-131 SPECT tumor imaging: Experimental measurements and photon transport simulations," IEEE Trans. Nucl. Sci., vol. 44, pp. 74-82, Feb. 1997.

[20] D. J. de Vries, S. C. Moore, R. E. Zimmerman, S. P. Mueller, B. Friedland, and R. C. Lanza, "Development and validation of a Monte Carlo simulation of photon transport in an anger camera," IEEE Trans. Med. Imaging, vol. 9, pp. 430-438, 1990.

[21] M. S. Rosenthal and L. J. Henry, "Scattering in uniform media," Phys. Med. Biol., vol. 35, pp. 265-274, 1990. 